\title{
Cutaneous B-Cell Pseudolymphoma Successfully Treated with Triamcinolone Acetonide
}

\author{
Tulus Dyah Anggraeni* ${ }^{\mathbb{D}}$, Airin Nurdin, Farida Tabri, Faridha llyas \\ Department of Dermatology and Venereology, Hasanuddin University, Makassar, South Sulawesi, Indonesia
}

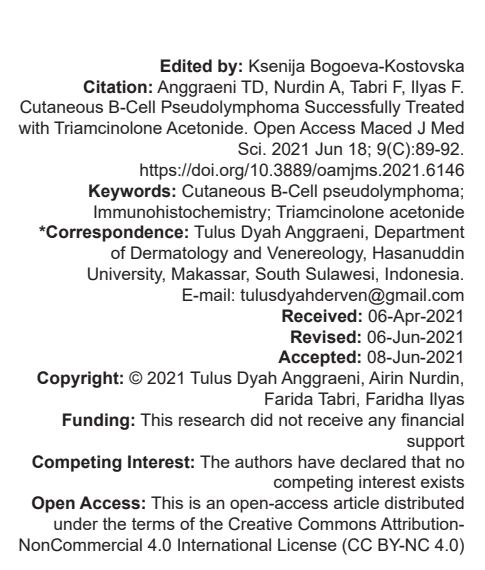

\begin{abstract}
BACKGROUND: Cutaneous pseudolymphoma (PSL) is a reactive polyclonal benign lymphoproliferative process in the skin that simulate cutaneous lymphomas clinically, histologically, or both, predominantly composed of either B-cells or T-cells, localized or disseminated. PSL clinically manifests as solitary nodules or plaque on the face. In cases where cutaneous PSL is suspected, the most crucial part is diagnosis, to differentiate benign or malignant lesion. Diagnosis required a combination of clinical, histopathological, and immunohistochemistry examination.

CASE REPORT: A 59-year-old man presented with asymptomatic erythematous plaque on her cheek for 6 months before. Histopathological examination revealed dense small lymphocytic infiltration forming lymphoid follicles with centrum germinativum that partially destructed skin appendice glands. Immunohistochemistry examination showed positive result on cluster of differentiation (CD)20 and CD3 staining. With domination of CD20 treatment: Patient was treated with intralesional injection of triamcinolone acetonide $10 \mathrm{mg} / \mathrm{ml}$ and showed satisfying result after 3 times injection.

CONCLUSION: A cutaneous B-cell PSL in a 59-year-old man was diagnosed based on history and physical, histopathological, and also immunohistochemistry examination. Intralesional injection of $10 \mathrm{mg} / \mathrm{ml}$ triamcinolone
\end{abstract} acetonide gave satisfying result.

\section{Introduction}

Cutaneous pseudolymphoma (PSL) is a term used for benign reactive lymphoproliferative skin disorders that mimic cutaneous lymphoma, both clinically and/or histopathologically. These diseases differ in clinical, histological, immunophenotypic presentation, and etiology [1], [2]. The term PSL was first introduced by Kaposi in 1891 and the term cutaneous PSL was coined by Burg et al. in 1982 [3].

Based on the dominant lymphocyte infiltrate, cutaneous PSL is classified into B-cell PSL (B-PSL), T-cell PSL (T-PSL), and mix PSL [4]. Cutaneous B-PSL often presents as nodule or plaque. Immunohistochemical staining shows that most infiltrates are more dominantly stained with B-cell markers than T-cell markers. Cells in reactive follicles express BCL-6 (+) and BCL-2 (-) [1].

Cutaneous PSL may affect all age group; Borrelia-induced B-PSL more commonly occurs in children and young adults while drug-induced T-PSL is more commonly encountered in adults. Even though Borrelia-induced PSL may be a precursor for B-cell neoplasms of the skin, in general, cutaneous PSL is self-limiting and does not affect survival [2].
Clinical features of cutaneous PSL include papules, plaques, or purplish erythema nodules, which are mostly found on the face [4], [5], [6]. A broad spectrum of known causative factors that may induce cutaneous PSL has been identified. Clinicopathological correlation is central in establishing the diagnosis of cutaneous PSL and distinguishing it from skin lymphoma. Infectious agents, such as spirochetal bacteria (Borrelia burgdorferi sp. and Treponema pallidum), viruses (e.g.: Parapoxvirus), and infestations (e.g. scabies), insect bites, injection of vaccines, or desensitization antigens, foreign bodies such as tattoos and metals, and drugs have been identified as causative factors for PSL. All cases without identifiable causes are called idiopathic PSL [1], [5], [6], [7].

Here, we report one case of cutaneous B-PSL in a 59-year-old man who was successfully treated with intralesional triamcinolone acetonide injection.

\section{Case Report}

A59-year-old man came to the Dermatovenerology Outpatient Clinic of Hasanuddin University Hospital, Makassar, Indonesia, with a chief complaint of redness lump 
on the left cheek that appeared 6 months before admission. The lump was originally in the form of skin-colored small nodules resembling acne that reddened, increased in number, and widened. The lumps were not painful nor itchy. The patient denied history of previous medication, insect bites, or trauma that preceded the complaint. Family history with similar complaints and history of diabetes were dined. History of food allergies and drug allergies was also absent.

Physical examination showed that vital signs were within normal limit. Lymph node enlargement was absent. The results of laboratory tests of complete blood count, hematologic, and blood chemistry (glucose, kidney function, and liver function) were within normal limits.

Dermatological examination revealed erythematous plaque with irregular edges on the left preauricular region (Figure 1a). On palpation, the plaque was compressible and fixed without any tenderness. The differential diagnosis included sarcoidosis, lupus vulgaris, leprosy, cutaneous PSL, and cutaneous lymphoma.

Biopsy examination was done to confirm the cause of the lesion. Histopathological examination showed normal epidermis and clusters of lymphocytes forming nodules in the dermis with some surrounded the adnexal structures (Figure 2). No atypical lymphocytes nor mitotic activity was found. Immunohistochemical staining showed cluster of differentiation (CD)3 and CD20 expression on the lymphocyte membrane, with CD20 being more dominant (Figure 3 and 4). These findings were consistent with that of cutaneous B-PSL.
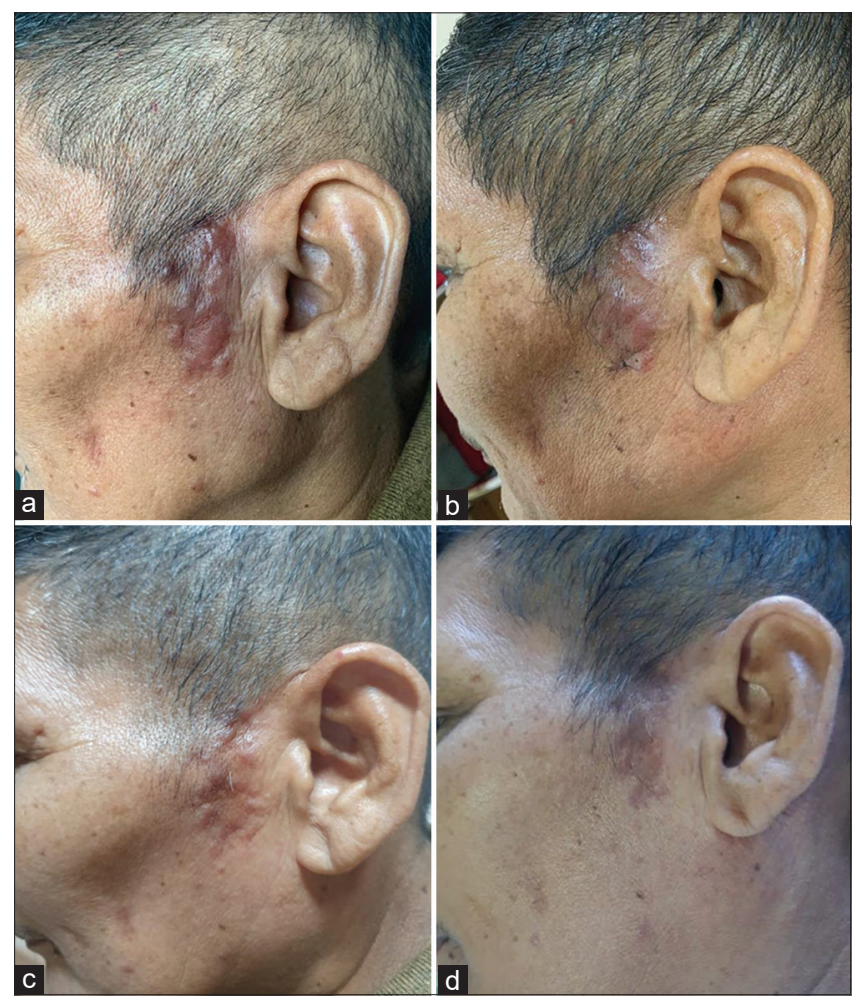

Figure 1: Left preauricular region. (a) Erythematous plaque with irregular edges on the first admission. (b) Thinning of the lesion 1 week after the first injection of triamcinolone acetone. ( $c$ and d) The plaques faded to brown and thinned 1 week after the second and third injection of triamcinolone acetonide
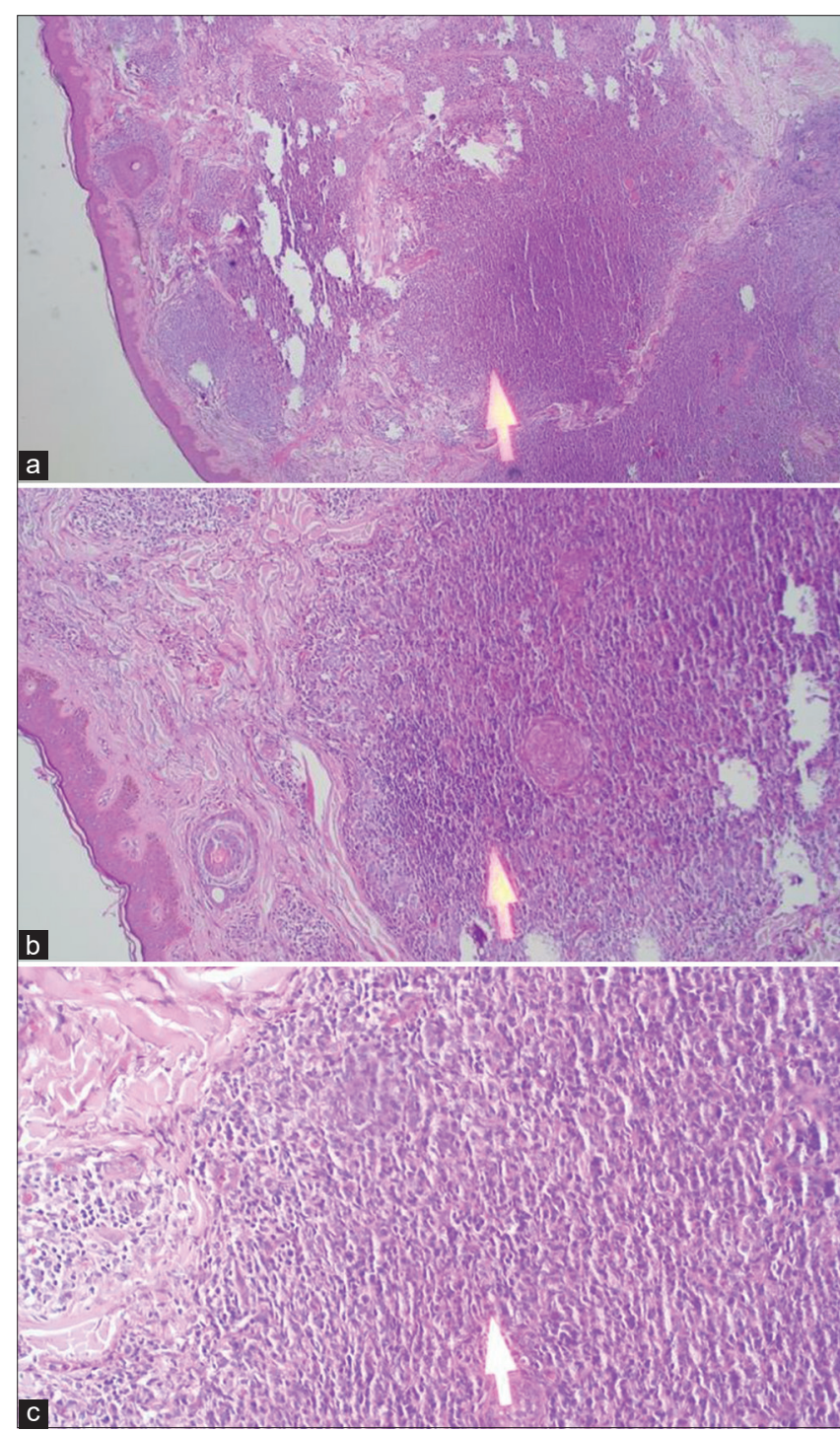

Figure 2: (a-c) Histopathological examination. (a) $\times 4$, HE staining, epidermis was still good, dermis showed distribution of lymphocyte cells arranged in groups to form nodules (nodular structure), some areas appear diffuse and compact, and appear to surround the adnexal structure, infiltrate consists of lymphocytes small, dense small lymphocyte form lymphoid follicles with centrum germinativum which partially degrades the skin's appendices, there were eosinophils and macrophages, lymphocytes do not show atypia, no mitotic activity was found. (b) $\times 10$, HE staining. (c) $\times 40$, HE staining, no mitotic activity was found

Based on the history, physical, and supporting examinations, the patient was diagnosed as B-PSL. Intralesional injection of $10 \mathrm{mg} / \mathrm{ml}$ triamcinolone acetonide per week was administered and the lesion showed significant improvement after three courses of injections (Figure $1 \mathrm{~b}$ and d). The erythematous nodules faded into brownish plaques (Figure 1c) and no side effects were observed.

\section{Discussion}

In cases of suspected cutaneous PSL, excluding the possibility of malignancy is of utmost 


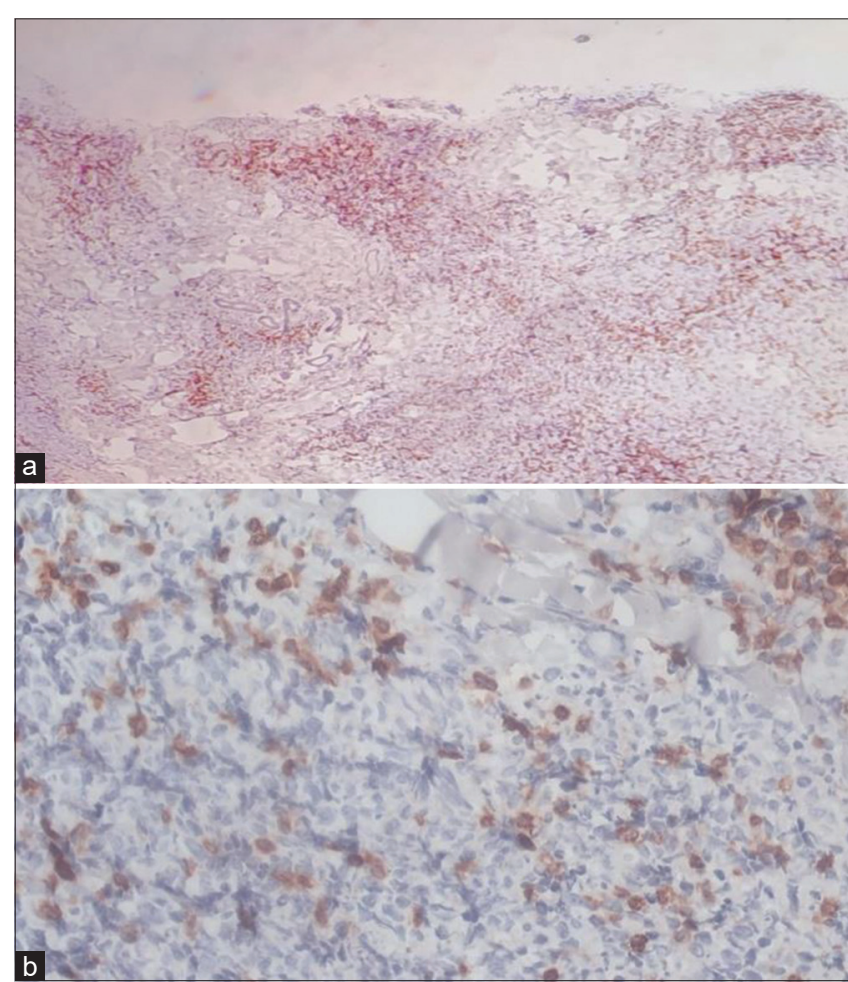

Figure 3: ( $a$ and b) Immunohistochemistry staining. Staining with cluster of differentiation (CD)3 in the epidermis and dermis. CD3 on CD-positive lymphocyte cell membrane 3: Receptor protein expressed in the membrane of some cells from lymphoid tissue (positive), (a) $\times 4$, (b) $\times 40$

importance [4], [8]. The diagnosis must always be made based on the combination of clinical and histopathological examination and often requires additional examinations such as immunohistochemistry and molecular biological examination [7], [9] .

Clinically, most PSL cases manifest as solitary erythematous to brown or purplish plaque with facial predilection; however, it can also appear as solitary to multiple papules or infiltrated plaques [4]. Some cases also reported unusual forms in the form of ulcerations and keloids [10], [11].

Based on the clinical and/or histological presentation, there are four main groups of cutaneous PSL: Nodular PSL, pseudomycosis fungoides, other PSL (representing different clinical entities), and intravascular PSL [12], [13]. In this case, dermatological examination revealed erythematous plaque with irregular edges on the left preauricular region which was consistent with nodular PSL.

Pathologically, cutaneous PSL is classified into ordinary PSL (O-PSL), PSL with dominant B-cell infiltrates (B-PSL), PSL with dominant T-cell infiltrates (T-PSL), and PSL with mixed and unclassified infiltrate [2], [7]. In immunohistochemical examination, most infiltrates are represented by more or dominant B-cell markers than T-cell markers [1].

Antigens in cutaneous PSL can be in the form of insect bites, tattoos, trauma, vaccinations, jewelry,

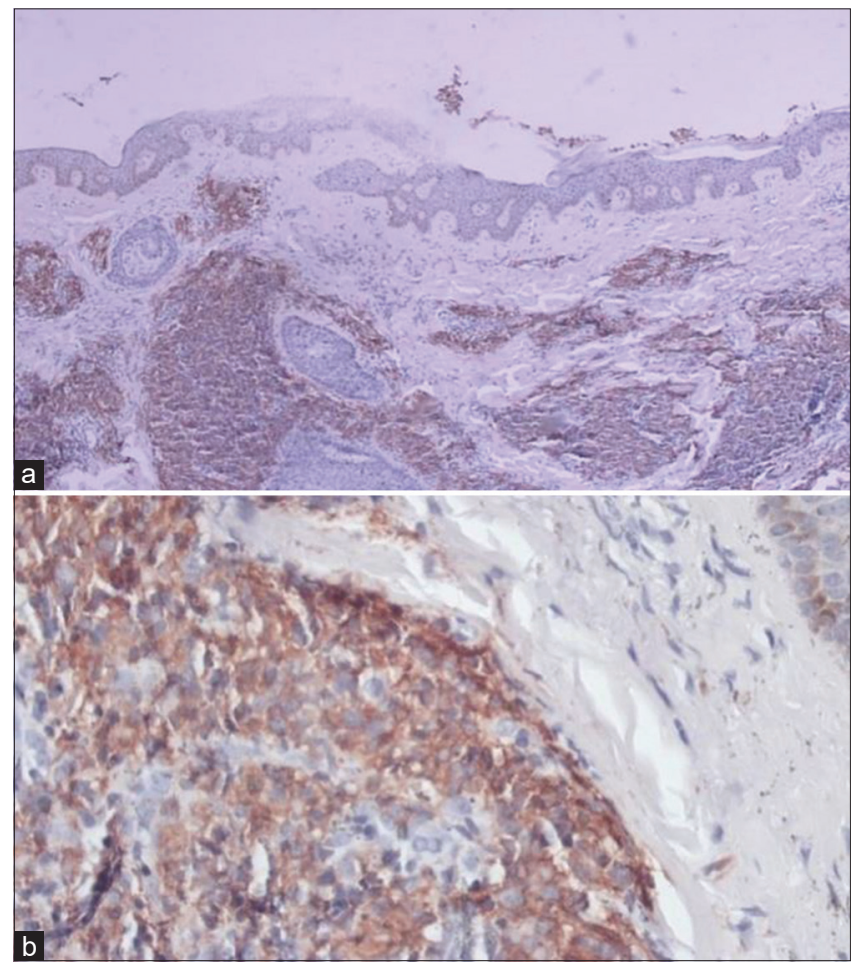

Figure 4: (a and b) Immunohistochemistry staining. Staining with cluster of differentiation (CD) 20 in the epidermis and dermis. CD20 on the lymphocyte cell membrane. CD20 positive: Receptor protein expressed in the membrane of some cells from lymphoid tissue (positive), (a) $\times 4$, (b) $\times 40$

drugs, and infection by $B$. burgdorferi. In many cases, the etiology of PSL cannot be unveiled and, therefore, can be classified as an idiopathic case [7], [8], [9].

In this case, no abnormalities were found in the epidermis and positive B-cell markers (CD20) as well as a rare cell population expressing T-cell markers (CD3) were shown by immunohistochemical staining. In cutaneous B-PSL, immunohistochemical study will usually reveal the predominance of B-cells with variable numbers of T-cells. Thus, a diagnosis of B-PSL could be established [1], [14]. Important histopathological feature of PSL is lymphoid proliferation with involvement of the centrum germinativum, infiltrate consisting of small lymphocytes, a group of small dense lymphocytes forming lymphoid follicles with germinativum centrum which partially degrades the appendices of the skin, and a minimal degree of atypia. Immunohistochemistry examination typically gives positive B-cell and T-cell marker, the bias can be seen in the presence of eosinophil, macrophages, histiocytes, and dendritic cells [4], [6], [9]. The involvement of centrum germinativum and polyclonality of lymphocytes will rule out a differential diagnosis of cutaneous lymphoma [4].

Treatment options for cutaneous PSL are determined based on the causative factor, area of the lesion, anatomic location, and patient's needs [10]. Spontaneous resolution is reported in some cases with cessation of the underlying etiology and those of idiopathic nature [10], [11]. Treatment options involve intralesional and systemic steroids, cryosurgery, 
alpha interferon, excision, local radiotherapy, and immunosuppressants [4], [5], [11]. In this case, as the lesion was solitary and localized, we decided to administer intralesional triamcinolone acetonide injection. Triamcinolone acetonide injection resulted in a significant improvement after the third injection and no side effects were found in the patient. In other reports, treatment with topical and/or intralesional corticosteroids resulted in improvement in the skin disease [3]. Lesions of PSL were treated with intralesional triamcinolone acetonide injection $10 \mathrm{mg} / \mathrm{ml}$ for 2 sittings, 3 weeks apart following which resulted in complete remission of the nodules without any relapse on follow-up [7].

It is important to understand that neither clinical nor histological features alone are sufficient to distinguish PSL from lymphoma. A combination of clinical signs, histological features, and the course of the disease is needed to obtain the correct diagnosis. Sometimes, a careful drug history, serological tests, and patch tests may help to distinguish CPL from lymphoma. Nevertheless, since progression of $\mathrm{CPL}$ to malignant lymphoma can occur, perhaps induced by persistent antigenic stimulation, regular follow-up of the patient is mandatory. However, progression to cutaneous lymphoma has been observed in only a minority of cases [3].

\section{Conclusion}

Cutaneous PSL is a term used for benign reactive lymphoproliferative skin disorders that mimic cutaneous lymphoma, both clinically and/or histopathologically. Therapy with triamcinolone acetonide injection of $10 \mathrm{mg} /$ $\mathrm{ml}$ resulted in a satisfactory outcome.

\section{References}

1. Kerns ML, Anna L, Kang S, Fitzpatrick's W, Kempf W, Stadler R, et al. Cutaneous pseudolymphoma. In: Kang S, Amagai M, Bruckner AL, Alexander H, Margolis DJ, McMichael AJ, et al.
Fitzpatrick's. $9^{\text {th }}$ ed. 2019.

2. Terada T. Cutaneous pseudolymphoma: A case report with an immunohistochemical study. Int J Clin Exp Pathol. 2013;6(5):966-72.

PMid:23638232

3. Miguel D, Peckruhn M, Elsner P. Treatment of cutaneous pseudolymphoma: A systematic review. Acta Derm Venereol. 2018;98(3):310-7.

PMid:29136262

4. Shetty SK, Hegde U, Jagadish L, Shetty C. Pseudolymphoma versus lymphoma: An important diagnostic decision. J Oral Maxillofac Pathol. 2016;20(2):328.

PMid:27601833

5. Bergman R. Pseudolymphoma and cutaneous lymphoma: Facts and controversies. Clin Dermatol. 2010;28(5):568-74. PMid:20797521

6. Koh WL, Tay YK, Koh MJ, Sim CS. Cutaneous pseudolymphoma occurring after traumatic implantation of a foreign red pigment. Singapore Med J. 2013;54(5):e100-1.

PMid:23716159

7. Shashikumar B, Harish M, Katwe K, Kavya M. Cutaneous pseudolymphoma: An enigma. Clin Dermatol Rev. 2017; $1: 22$

8. Prabhu V, Shivani A, Pawar V. Idiopathic cutaneous pseudolymphoma: An enigma. Indian Dermatol Online J. 2014;5(2):224-6.

PMid:24860772

9. Hussein MR. Cutaneous pseudolymphomas: Inflammatory reactive proliferations. Expert Rev Hematol. 2013;6(6):713-33.

PMid:24191857

10. Magro $\mathrm{CM}$, Daniels $\mathrm{BH}$, Crowson AN. Drug induced pseudolymphoma. Semin Diagn Pathol. 2018;35(4):247-59. PMid:29361381

11. Bombonato C, Pampena R, Lallas A, Giovanni P, Longo C. Dermoscopy of lymphomas and pseudolymphomas. Dermatol Clin. 2018;36(4):377-88 PMid:30201147

12. Mitteldorf C, Kempf W. Cutaneous pseudolymphoma. Surg Pathol Clin. 2017;10(2):455-76. PMid:28477891

13. Mitteldorf C, Kempf W. Cutaneous pseudolymphoma-a review on the spectrum and a proposal for a new classification. J Cutan Pathol. 2020;47(1):76-97.

PMid:31237707

14. Sasidharanpillai S, Aravindan K, Nobin B, Raghavan N, Nikhila P, Riyaz N. Phenytoin induced cutaneous B cell pseudolymphoma. Indian J Dermatol. 2015;60(5):522.

PMid:26538730 